\title{
ATYPICAL DUST SPECIES IN THE EJECTA OF CLASSICAL NOVAE
}

\author{
L.A. Helton ${ }^{1}$, A. Evans ${ }^{2}$, C.E. Woodward ${ }^{1}$ and R.D. Gehrz ${ }^{1}$
}

\begin{abstract}
A classical nova outburst arises from a thermonuclear runaway in the hydrogen-rich material accreted onto the surface of a white dwarf in a binary system. These explosions can produce copious amounts of heavy element enriched material that are ejected violently into the surrounding interstellar medium. In some novae, conditions in the ejecta are suitable for the formation of dust of various compositions, including silicates, amorphous carbon, silicon carbide, and hydrocarbons. Multiple dust grain types are sometimes produced in the same system. CO formation in novae may not reach saturation, thus invalidating the usual paradigm in which the $\mathrm{C}: \mathrm{O}$ ratio determines the dust species. A few novae, such as V705 Cas and DZ Cru, have exhibited emission features near 6,8 , and $11 \mu \mathrm{m}$ that are similar to "Unidentified Infrared" (UIR) features, but with significant differences in position and band structure. Here, we present Spitzer IRS spectra of two recent dusty novae, V2361 Cyg and V2362 Cyg, that harbor similar peculiar emission structures superimposed on features arising from carbonaceous grains. In other astronomical objects, such as star forming regions and young stellar objects, emission peaks at 6.2, 7.7, and $11.3 \mu \mathrm{m}$ have been associated with polycyclic aromatic hydrocarbon (PAH) complexes. We suggest that hydrogenated amorphous carbon (HAC) may be the source of these features in novae based upon the spectral behavior of the emission features and the conditions under which the dust formed.
\end{abstract}

\section{Introduction}

A classical nova (CN) is an eruption arising on the surface of a white dwarf (WD) in a binary system in which the WD is accreting material from a main sequence

\footnotetext{
1 Department of Astronomy, School of Physics and Astronomy, University of Minnesota, 116 Church St. SE, Minneapolis, MN 55455, USA; e-mail: ahelton@astro.umn.edu; chelsea@astro.umn.edu; \& gehrz@astro.umn.edu 2 Astrophysics Group, Keele University, Keele, Staffordshire ST5 5BG, UK; e-mail: ae@astro.keele.ac.uk
} 
companion through Roche-lobe overflow. The accreted material accumulates on the surface of the WD until it reaches a density and temperature high enough to initiate a thermonuclear runaway (TNR), resulting in explosive nucleosynthesis and violent ejection of up to $10^{-6}$ to $10^{-4} M_{\odot}$ of accreted material at velocities exceeding $1000 \mathrm{~km} \mathrm{~s}^{-1}$. The ejecta are initially optically thick, but as they expand, the density rapidly declines resulting in the recession of the optically thick pseudophotosphere inwards towards the hot underlying WD. This energetic underlying central engine actively photoionizes the ejecta, resulting in the appearance of emission lines from a wide range of isoelectronic species.

CNe frequently produce dust in their ejecta. The dust condensation event is signaled by a rapid decline in the optical light curve due to extinction and a corresponding rise in the infrared luminosity due to thermal emission. Models of the spectral energy distribution (SED) in these systems suggest that the condensation event yields relatively large dust grains with radii up to $\sim 1 \mu \mathrm{m}$ (Gehrz 2008). Unlike most astronomical sources of dust, novae have been observed to produce both carbon rich and oxygen rich dust species simultaneously (e.g., Gehrz et al. 1992). Evans \& Rawlings (2008) suggest that this chemical dichotomy may be explained by incomplete carbon monoxide formation in the ejecta. If $\mathrm{CO}$ does not form to saturation, then neither carbon nor oxygen will be entirely bound up in $\mathrm{CO}$, leaving both available for dust production.

\section{Atypical dust species in classical novae}

Novae V705 Cas, V842 Cen, and QV Vul exhibited emission features similar to unidentified infrared features (UIRs; Geballe 1997) often attributed to polycyclic aromatic hydrocarbons (PAHs; Allamandola et al. 1989) or hydrogenated amorphous carbons (HACs; Duley \& Williams 1983). Observations of V705 Cas beginning 157 days after outburst revealed UIR features at 8.2, 8.7, and $11.4 \mu \mathrm{m}$ superimposed on a $10 \mu \mathrm{m}$ silicate emission feature (Evans et al. 1997). Subsequent analysis of the $8 \mu \mathrm{m}$ complex by Evans et al. (2005) indicated that the band shape and peak wavelength did not match those of standard UIR features and that the peak wavelength drifted bluewards as the system evolved. Simultaneously, the ratio of the 11.4 to $8 \mu \mathrm{m}$ features increased. The $3-4 \mu \mathrm{m}$ region in the spectrum of V705 Cas showed emission features at 3.28 and $3.4 \mu \mathrm{m}$. These are typically attributed to unsaturated $\mathrm{C}-\mathrm{H}$ stretching mode oscillations in aromatic molecules and saturated $\mathrm{C}-\mathrm{H}$ stretching vibrations in aliphatic bonds, respectively. Thus, the $3.28 / 3.4 \mu \mathrm{m}$ ratio can be used as an indicator of the relative abundance of aromatic to aliphatic structures in the emitting material (Sloan et al. 1997). The observed 3.28 to $3.4 \mu \mathrm{m}$ ratio was similar to that seen in post-AGB stars, but an order of magnitude higher than in stars with a high UV photon flux. The behavior of these features in V705 Cas was similar to those observed in V842 Cen and QV Vul. Evans \& Rawlings (1994) suggested that during the optically thick stage of dust formation, the rate of $\mathrm{H}$ capture is greater than the rate of $\mathrm{UV}$ photon absorption. Thus, the conditions are conducive to the growth of aliphatic bonds, e.g. HAC grains. 

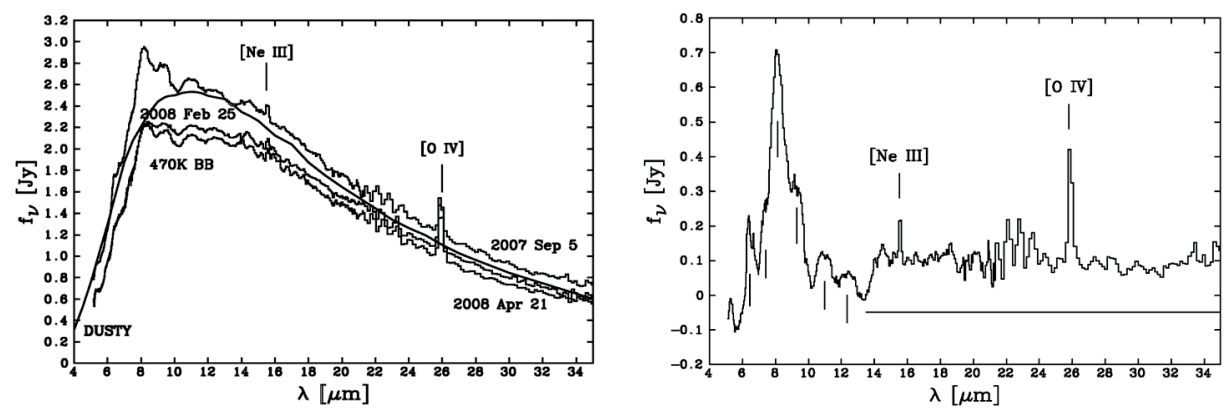

Fig. 1. Left: Spitzer IRS spectra of DZ Cru with a DUSTY model of AC at $470 \mathrm{~K}$. Right: residuals after DUSTY model subtraction showing UIR features.

\section{$2.1 \quad D Z C r u$}

DZ Cru was observed as part of our Spitzer Classical Novae Target-of-Opportunity and Monitoring campaign (Evans et al. 2010). Due to poor sampling of the light curve, the optical depth of the dust extinction event is not known. Our first Spitzer Infrared Spectrograph (IRS) observations commenced nearly 1500 days after eruption. They revealed exceptionally strong emission from amorphous carbon (AC) with superimposed UIR emission features reminiscent of those observed in V705 Cas. The left panel of Figure 1 shows three epochs of Spitzer IRS data along with a DUSTY (Ivezić et al. 1999) model of AC dust at $470 \mathrm{~K}$. Subtraction of the DUSTY model (Fig. 1, right panel) revealed emission features at 6.5, 7.2, 8.1, 9.3, 11.0, and $12.4 \mu \mathrm{m}$. The wavelengths of these features do not correspond with the standard PAH features observed at $6.2,7.7,8.6,11.3$, and $12.7 \mu \mathrm{m}$.

\subsection{V2362 Cyg}

V2362 Cyg was a peculiar nova in many respects (see Lynch et al. 2008). One of the most interesting characteristics was its optical light curve behavior. After a smooth initial decline the light curve plateaued and subsequently experienced a secondary brightening event, which rivaled its initial outburst luminosity. At the peak of the second outburst, the nova underwent a rapid and extreme dust formation event. The Spitzer IRS spectra obtained immediately following dust condensation revealed blackbody emission at $1400 \mathrm{~K}$, near the extreme high end of dust condensation temperatures. At first, low ionization [Ne II], [Ne III], and [O IV] were observed in the spectra alongside a hydrogen recombination spectrum. But within a few days, as the dust emission strengthened, the emission lines disappeared with the sole exception of [O IV], which remained strong throughout.

With the decline in the dust optical depth due to expansion of the ejecta, the emission lines reappeared, but at a much higher ionization level. A range of ionization states was observed including [Ne III], [Ne V], [Ne VI], [Mg V], and $[\mathrm{Mg}$ VII], indicating a very energetic ionization field. Indeed, Swift observations 
from this period indicated a strong, hard X-ray flux that persisted for at least 300 days.

During this same period of optically thin dust emission, strong, broad UIR features near $6-10,11.5$, and $18 \mu \mathrm{m}$ became evident. We fitted and subtracted a blackbody continuum to isolate the emission components. The results are shown in the left panels of Figure 2. Distinct emission peaks appeared at 6.4, 6.9, 7.9, $9.5,11.4,12.5$, and $17.9 \mu \mathrm{m}$. The features near 9.5 and $18 \mu \mathrm{m}$ may be associated with silicates. As the ejecta evolved, the central wavelengths of the UIR features varied slightly and irregularly, while the flux ratios changed dramatically. The $6.9,11.4$, and $12.5 \mu \mathrm{m}$ features declined rapidly and soon disappeared. The fluxes of the 6.4 and $7.9 \mu \mathrm{m}$ features declined sharply relative to the 9.5 and $17.9 \mu \mathrm{m}$ features. The $17.9 \mu \mathrm{m}$ feature began to dominate the spectrum at late times.

\subsection{V2361 Cyg}

The spectrum of V2361 Cyg was very similar to that of V2362 Cyg. The primary differences were the lack of emission features at 9.5 and $18 \mu \mathrm{m}$ and additional structure in the 6-10 $\mu \mathrm{m}$ spectral region. Spectra obtained as part of our Spitzer program were fitted with blackbody curves. The residuals after blackbody subtraction are shown in the right panels of Figure 2. UIR emission features were observed at $5.4,6.4,6.9,7.1,7.5,8.1,11.5$, and $12.4 \mu \mathrm{m}$. As the ejecta evolved, the complex of features between 6.4 and $8.1 \mu \mathrm{m}$ blended together to form a single plateau, while the 6.4 to $8.1 \mu \mathrm{m}$ flux ratio diminished significantly. The 11.5 and $12.4 \mu \mathrm{m}$ features joined to produce a broad structure around $13.1 \mu \mathrm{m}$.

In Figure 2-h, we compare the observed emission from V2361 Cyg at 102 days post outburst to the Class A, B, and C PAH features described by Peeters et al. (2002) and van Diedenhoven et al. (2004). The Class A and B PAHs may be excluded as potential contributors due to the poor fit to the emission peaks. The Class $\mathrm{C}$ profiles are more consistent with the data. In particular, the $8 \mu \mathrm{m}$ component of the Class $\mathrm{C}$ emission fits the red side of the profile quite well. Sloan et al. (2007) have attributed the Class C profiles to carriers with a high fraction of aliphatic bonds.

\section{The model}

The Spitzer IRS observations of DZ Cru, V2362 Cyg, and V2361 Cyg in combination with the previous studies of V705 Cas, V842 Cen, and QV Vul provide the basis for an elementary model for the processing of dust and complex hydrocarbon molecules in classical novae. The dust formation event proceeds quite rapidly with dust grains condensing out of the gas phase and growing to sizes up to $\sim 1 \mu \mathrm{m}$ in a matter of days. In part, this is likely due to the inhomogeneous, clumpy distribution of the ejecta. With the onset of grain formation, the opacity in these regions increases, shielding the rest of the material from the underlying radiation and allowing the acceleration of condensation. 

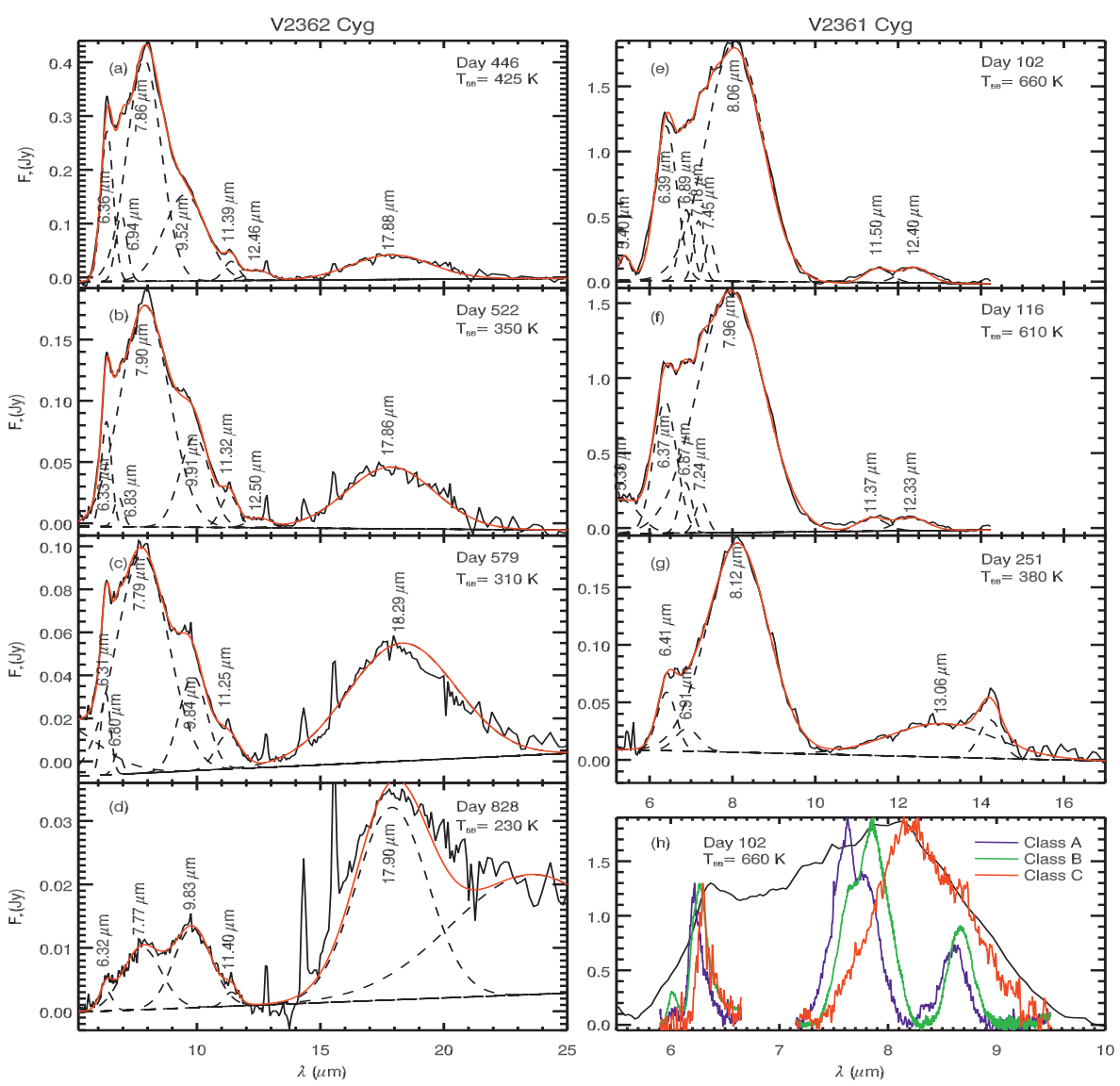

Fig. 2. Residuals for V2362 Cyg, (a)-(d), and V2361 Cyg, (e)-(g). Dashed lines are individual Gaussian components of the composite fit shown in red. Panel (h) shows Class A, B, and C PAH profiles (Peeters et al. 2002) overlayed on the V2361 Cyg spectra from Day 102.

The types of dust produced during the condensation event depend on the conditions and abundances in the ejecta. In typical novae, the dust formed is composed of AC, silicates, or some combination thereof. In the novae discussed here, the dominant dust species appears to be carbonaceous. Evans \& Rawlings (1994) argued that at the opacities observed during the dust condensation stage in novae, the likelihood for $\mathrm{H}$ capture is much higher than the probability of UV photon interaction, thus enhancing the production of aliphatic bonds. This conclusion suggests that the smaller end of the grain size distribution may be due to PAH molecules and larger, more complex HAC agglomerations. However, as the ejecta expand and densities decline, the optical depth of the dust forming regions decreases as the pseudophotosphere recedes, exposing the freshly condensed dust to an increasingly hard and intense radiation field. 
Evans \& Rawlings (1994) have calculated that small PAHs (i.e., molecules with fewer than $24 \mathrm{C}$ atoms) have a very short lifetime in the environment of a $\mathrm{CN}$. Due to the high UV flux, a free-flying PAH molecule would absorb about one high energy photon every second and would be completely disrupted in less than a day. These same molecules would also be subjected to chemical erosion through collisional interactions in the ejecta. For reasonable densities, temperatures, and abundances in the ejecta, Evans \& Rawlings estimated that these PAHs would be destroyed due to chemi-sputtering by $\mathrm{H}$ and $\mathrm{O}$ in a matter of hours.

If some of the emission features seen in V2361 Cyg, V2362 Cyg, and DZ Cru can be attributed to small PAHs instead of HACs, then there must be a reservoir of material from which the PAHs are being repopulated. This material could be produced by either HACs or the larger AC grains themselves when submitted to energetic processes. Though rapidly destroyed, the transient population of freeflying PAHs could generate some of the observed UIR emission features.

In order to better constrain the carriers of the UIR features observed in CN, it is critical to obtain observations of the expanding ejecta throughout the dust condensation and subsequent stages. If the carrier molecules are being formed during the initial condensation event, they may be observable during or immediately after condensation. Data covering the $3-4 \mu \mathrm{m}$ region are required in order to determine the relative contributions of aromatic to aliphatic material.

This work was supported in part by Spitzer/JPL grants 1294825 and 1289430 .

\section{References}

Allamandola, L.J., Tielens, A.G.G.M., \& Barker, J.R., 1989, ApJS, 71, 733

Duley, W.W., \& Williams, D.A., 1983, MNRAS, 205, 67P

Evans, A., \& Rawlings, J.M.C., 1994, MNRAS, 269, 427

Evans, A., Geballe, T.R., Rawlings, J.M.C., Eyres, S.P.S., \& Davies, J.K., 1997, MNRAS, 292, 192

Evans, A., Tyne, V.H., Smith, O., et al., 2005, MNRAS, 360, 1483

Evans, A., \& Rawlings, J.M.C., 2008, in Classical Novae, $2^{\text {nd }}$ Edition, ed. M.F. Bode \& A. Evans (Cambridge Univ. Press: Cambridge)

Evans, A., Gehrz, R.D., Woodward, C.E., et al., 2010, MNRAS, 406, 85

Geballe, T.R., 1997, From Stardust to Planetesimals, 122, 119

Gehrz, R.D., 2008, in Classical Novae, $2^{\text {nd }}$ Edition, ed. M.F. Bode \& A. Evans

(Cambridge Univ. Press: Cambridge)

Gehrz, R.D., 1992, ApJ, 400, 671

Ivezić, Ž., Nenkova, M., \& Elitzer, M., 1999 [arXiv:astro-ph/9910475]

Lynch, D.K., Woodward, C.E., Gehrz, R.D., et al., 2008, AJ, 136, 1815

Peeters, E., Hony, S., Van Kerckhoven, C., et al., 2002, A\&A, 390, 1089

Sloan, G.C., Jura, M., Duley, W.W., et al., 2007, ApJ, 664, 1144

Sloan, G.C., Bregman, J.D., Geballe, T.R., Allamandola, L.J., \& Woodward, C.E., 1997, ApJ, 474, 735

van Diedenhoven, B., Peeters, E., Van Kerckhoven, C., et al., 2004, ApJ, 611, 928 